\title{
Genome-wide Association Study and Possible Candidate Genes for Root Color and Carotenoid Contents in Japanese Orange Carrot F2 Populations
}

Taeko Shibaya ( $\sim$ komai@kazusa.or.jp )

Fujii Seed Co. Ltd

Chika Kuroda

Fujii Seed Co. Ltd

Hisano Tsuruoka

Kazusa DNA Research Institute

Chiharu Minami

Kazusa DNA Research Institute

Akiko Obara

Kazusa DNA Research Institute

Shinobu Nakayama

Kazusa DNA Research Institute

Takayoshi Fujii

Fujii Seed Co. Ltd

Sachiko Isobe

Kazusa DNA Research Institute

\section{Research Article}

Keywords: Carrot , provitamin A, genome-wide association study (GWAS), single-nucleotide polymorphisms (SNPs)

Posted Date: November 10th, 2021

DOI: https://doi.org/10.21203/rs.3.rs-1014140/v1

License: (c) (1) This work is licensed under a Creative Commons Attribution 4.0 International License.

Read Full License 


\section{Abstract}

Carrot is a major source of provitamin A in a human diet. Two of the most important traits for carrot breeding are carotenoid contents and root color. To examine genomic regions related to these traits and develop DNA markers for carrot breeding, we performed a genome-wide association study (GWAS) using genome-wide single-nucleotide polymorphisms (SNPs) in two F2 populations, both derived from crosses of orange root carrots bred by a Japanese seed company. The GWAS revealed 21 significant associations, and the physical position of some associations suggested two possible candidate genes. An Orange (Or) gene was a possible candidate for visual color evaluation and the $\alpha$ - and $\beta$-carotene contents. Sanger sequencing detected a new allele of Or with an SNP which caused a non-synonymous amino acid substitution. Genotypes of this SNP corresponded to the visual evaluation of root color in another breeding line. A chromoplast-specific lycopene $\beta$-cyclase (CYC-B) gene was a possible candidate for the $\beta / \alpha$ carotene ratio. On CYC-B, five amino acid substitutions were detected between parental plants of the F2 population. The detected associations and SNPs on the possible candidate genes will contribute to carrot breeding and the understanding of carotenoid biosynthesis and accumulation in orange carrots.

\section{Introduction}

Carrot (Daucus carota L.), a major source of provitamin A carotenes in the human diet, is consumed worldwide [1]. Carrots accumulate abundant carotenoids in their taproots, and these carotenoids (which are responsible for the orange pigmentation in the carrot roots) are thought to provide health benefits [2]. A variety of colors has been observed in carrot taproots, including orange, white, yellow, red, and purple. Quantitative trait loci (QTL) analyses and association studies for carrots' root color and carotenoid contents have been performed in several populations, and important and useful QTLs have been reported [3-6]. These studies used populations derived from crosses between accessions showing clearly different root colors such as orange and white [3-5] and orange and dark orange [4], and other studies used inter-crossed populations derived from white, yellow, red, and orange carrots [6].

Carotenoid biosynthesis is well established, and a highly conserved carotenoid biosynthesis pathway has been characterized in many plant species (Fig. 1) [7-9]. In carrot, several carotenoid biosynthetic genes have been mapped [3], and the released carrot whole-genome sequences showed orthologous and homologous genes involved in the carotenoid biosynthesis pathway $[10,11]$. Several genes involved in carotenoid biosynthesis and accumulation in carrot have also been identified. An ortholog of carotene hydroxylase CYP97A3 in the carotenoid biosynthesis pathway has been identified in carrot; it controls the $a$-carotene, total carotenoid contents, and the $\alpha / \beta$ carotene ratio [12]. A candidate gene association study of the carotenoid biosynthesis pathway revealed associations between the total carotenoid and $\beta$ carotene contents and the genes zeaxanthin epoxidase (ZEP), phytoene desaturase (PDS), and carotenoid isomerase (CRTISO), between the a-carotene content and the genes CRTISO and plastid terminal oxidase (PTOX), and between color components and the gene ZEP [6]. 
It was also reported that not only genes in the carotenoid biosynthesis pathway but also genes that have other functions considerably affect carotenoid contents. $Y$ and $Y_{2}$ loci account for most of the color differences of orange, yellow, and white carrot roots [13]. The $Y$ gene has been identified, and this gene has been hypothesized to regulate photosystem development and functional processes, including photomorphogenesis and root de-etiolation [10]. The $Y 2$ locus has been mapped to an approx. 650-kb genomic region; in addition, no annotated gene involved in the carotenoid biosynthesis pathway was located within the candidate region [14]. An Orange(Or) gene, which was first identified in cauliflower and accounted for an abnormally elevated $\beta$-carotene accumulation [15], was identified in carrot and is associated with the presence of carotenoid in carrot [16]. However, the genes, polymorphisms, and genomic regions involved in carotenoid biosynthesis and the carotenoid accumulation that cause slight differences in root color and carotenoids are not fully understood, especially within the orange carrots.

In Japan, consumers prefer a bright orange root color for carrots, and a cultivar showing uniform root colors is popular. There are accessions showing slight color differences in bright orange roots, and breeders in Japan have selected the best 'bright orange' and uniform color among the accessions that have bright orange roots. DNA markers that can be used to distinguish slight differences within bright orange color have thus been sought in Japanese carrot breeding. Toward this goal, there has been no study using populations derived from a cross between orange root carrots with slight color differences, but the recent release of whole genome sequences of carrot has made it easier to analyze whole-genome constitutions with high marker density and to conduct association analyses, even in the populations derived from genetically close orange carrots $[10,11]$.

In the present study, we developed two $F_{2}$ populations that have a common parent. Both populations were derived from crosses between orange-root parents. We performed a genome-wide association study (GWAS) to investigate the genomic regions that cause slight but important differences in the root color and carotenoid contents within carrots with orange root color.

\section{Methods}

\section{Plant materials}

We developed two $F_{2}$ populations ( $A$ and $B$ ) using orange-colored carrot plants bred by a Japanese seed company, Fujii Seed (Osaka, Japan). Population A was derived from a cross between Fs001 and Fs002, and population B was derived from a cross between Fs002 and Fs003 (Fig. 2). Fs002 was the pollen parent for $F_{2}$ population $A$ and the seed parent for $F_{2}$ population B. Plants of $F_{2}$ populations $A(n=146)$ and $B(n=136)$ were cultivated from mid-February to early June 2018 in a natural field at Narashino, Chiba, Japan, and used for DNA extraction and the visual evaluation of root colors. Roots of population A were also used for the quantification of carotenoid content by high-performance liquid chromatography (HPLC) and the measurement of color components. 
To examine a developed DNA marker on Orgene, we also used breeding line $\mathrm{C}$, which was bred by Fujii Seed. This line was developed by using Fs002 as one of the breeding materials (Fig. 2). Breeding line $\mathrm{C}$ was cultivated from the end of March to early July 2017 in a natural field at Oirase, Aomori, Japan, and 40 plants were used for DNA extraction and the visual evaluation of root colors.

Experimental research and field studies on plant materials comply with relevant institutional, national, and international guidelines and legislation.

\section{Visual evaluation of root colors and evaluation of color components}

The visual evaluation of root colors was performed by two experienced breeders at Fujii Seed. The root colors were visually evaluated to ten grades of orange darkness in $F_{2}$ population $A$, and to seven grades in $F_{2}$ population $B$, and to three grades in breeding line $C$. In the $F_{2}$ population $A$, color components ( $L^{*}, a^{*}$ and $b^{\star}$ ) were measured with a spectrocolorimeter (model CM2600d, Minolta, Tokyo) equipped with a 5$\mathrm{mm}$ measuring area. The surface of the middle part of washed carrot root was measured three times, and the average values were used for phenotypic data.

\section{Quantification of carotenoid contents (a-carotene, $\beta$ - carotene, and lutein) by HPLC}

Carrot root surface, i.e., approx. 1-2 mm of outer parts from phloem in the middle of roots was cut and collected. The collected samples were immediately frozen in liquid nitrogen and stored at $-80^{\circ} \mathrm{C}$. Root surface was used for HPLC because the visual and color component evaluations were performed on the carrot root surface. The extraction for HPLC was performed as described [6] with a scale-down and some modifications. Frozen samples were crushed into a powdery status with a tube mill control (S001, IKA, Staufen, Germany). Extraction was done on approx. $50 \mathrm{mg} \mathrm{(50} \mathrm{mg} \pm 5 \%$ ) of crushed frozen material to which $50 \mu \mathrm{L}$ of b-apo-8'-carotenal at $5 \mu \mathrm{g} / \mathrm{mL}$ was first added as an internal standard. Samples were mixed with $600 \mu \mathrm{L}$ of $\mathrm{MgCO}_{3} 0.57 \%$, 3,5-di-tert-butyl-4-hydroxytoluene (BHT) $0.1 \%$ in methanol, then vortexed, and mixed with $600 \mu \mathrm{L}$ of $0.1 \% \mathrm{BHT}$-containing chloroform. After 10 times of vertical mixing and incubation for $15 \mathrm{~min}$ in darkness at $4^{\circ} \mathrm{C}, 600 \mu \mathrm{L}$ of ultrapure water was added, and samples were centrifuged at $236 \mathrm{~g}$ for $10 \mathrm{~min}$. Next, $400 \mu \mathrm{L}$ from the lower layer was concentrated under vacuum evaporation, and the dry extract was dissolved in $50 \mu \mathrm{L}$ of acetone containing $0.1 \% \mathrm{BHT}$. Samples were kept at $4^{\circ} \mathrm{C}$ and protected from direct light during the entire procedure.

The carotenoid quantification was done on an Ultimate 3000 HPLC system coupled with a diode array detector (Thermo Fisher Scientific, Waltham, MA, USA) according to the manufacturer's instruction with slight modifications. Carotenoids were separated on an Acclaim C30 column $(150 \times 2.1 \mathrm{~mm}, 3 \mu \mathrm{m}$, Thermo Fisher Scientific). The mobile phases were acetonitrile as eluent A, methanol/acetic ether (1:1, 
$\mathrm{v} / \mathrm{v}$ ) as eluent $\mathrm{B}$, and $10 \mathrm{mM}$ formic acid $(\mathrm{pH} 3.0)$ as eluent $\mathrm{C}$. The elution program was as follows: the proportions of solvent $A, B$ and $C$ were $85 \% A, 14.5 \% B$, and $0.5 \% C$ at $0-2$ min; $85 \%-44.5 \% A, 14.5 \%-$ $55 \% \mathrm{~B}$, and $0.5 \% \mathrm{C}$ at $2-7 \mathrm{~min} ; 44.5 \% \mathrm{~A}, 55 \% \mathrm{~B}$, and $0.5 \% \mathrm{C}$ at $7-21 \mathrm{~min}$; and returned to the initial conditions $(85 \% \mathrm{~A}, 14.5 \% \mathrm{~B}$, and $0.5 \% \mathrm{C})$ at $21.1-28.5 \mathrm{~min}$. The flow rate was $0.4 \mathrm{~mL} / \mathrm{min}$. The injection volume of the filtered sample by a $0.22-\mu \mathrm{m}$ PTFE membrane filter was $3.9 \mu \mathrm{L}$. Analytes were detected by a photodiode array detector at $450 \mathrm{~nm}$. The data were analyzed using Chromeleon 7 software (Thermo Fisher Scientific) based on internal calibration using b-apo-8'-carotenal and the extraction yield.

\section{Double-digest restriction site-associated DNA sequencing (ddRAD-seq) and GWAS}

Total genomic DNA was extracted from young leaves of carrot plants with the DNeasy Plant Mini Kit (Qiagen, Hilden, Germany). A double-digest restriction site-associated DNA sequencing (ddRAD-seq) analysis was performed as described [17] with the restriction enzymes Pstl and Mspl. The ddRAD-seq libraries were constructed and sequenced on a HiSeq 4000 platform (Illumina, San Diego, CA) in pairedend 101-nucleiotide (nt) mode as described [17]. Primary data processing such as deleting low-quality bases and trimming adapters, mapping onto carrot genome Daucus carota v2.0 [10], and filtering singlenucleotide polymorphisms (SNPs) to obtain high-confidence SNPs were performed as described [18]. The association analysis between the phenotype data and the genotype data was performed using the generalized linear model (GLM) of trait analysis by association, evolution, and linkage (TASSEL) ver. 5.2.40 [19].

\section{Sanger sequencing of candidate genes}

For the comparison of the genomic sequences of possible candidate genes between parental plants in $\mathrm{F}_{2}$ populations A and B, we performed Sanger sequencing from the start codon to the stop codon on the genes. The primers used in the Sanger sequencing are listed in Supplementary Table S1.

\section{SNP genotyping with KASP marker}

KASP marker, which genotypes an SNP on Orgene in this study, was developed and performed according to the manufacturer's instructions (Biosearch Technologies, Novato, CA).

\section{Phylogenetic analysis}

The sequences of LCYE, LCYB, CYC-B, NSY, and CCS in several reported plant species such as Solanum lycopersicum, Carica papaya, Citrus sinensis, Capsicum annuum, and Lillium lancifolium [20-23], Arabidopsis [7], and carrot [24] were obtained from the public databases NCBI 
(http://www.ncbi.nlm.nih.gov) and Phytozome (https://phytozome.jgi.doe.gov/pz/portal.html\#). We used CLUSTALW [25] to align the amino acid sequences and constructed the phylogenetic tree by using the neighbor-joining method [26] provided by MEGA X [27].

\section{Results}

\section{GWAS for the visual evaluation of root color and evaluations of color components and carotene contents in roots of $F_{2}$ populations $A$ and $B$}

$F_{2}$ populations $A$ and $B$ both showed a normal distribution in all root color evaluations (Suppl. Fig. S1), suggesting the involvement of multiple associations in carrot root color. The ddRAD-seq analysis detected 3,106 and 1,901 high-confidence SNPs in $F_{2}$ populations $A$ and $B$, respectively. The GWASs were performed using these genotypic data and values from the visual evaluation and evaluations of the color components and carotene contents in the carrot roots. In $\mathrm{F}_{2}$ population $\mathrm{A}$, significant associations were detected for the visual evaluation of root color (Fig. 3a); color components a* (Fig. 3c) and b* (Fig. 3d); acarotene (Fig. 3e), $\beta$-carotene (Fig. 3f), and lutein contents (Fig. 3g); and the $\beta /$ a-carotene ratio (Fig. $3 \mathrm{~h}$ ) in root (Table 1). No significant associations were detected for color component L* (Fig. 3b).

The associations for visual evaluation, color components $a^{*}$ and $b^{\star}$, and $a$ - and $\beta$-carotene contents on chromosome 1 were detected at close physical positions, and the highest associations were detected at a physical position around $31 \mathrm{Mb}$ (Fig. 3, Table 1), suggesting that these associations are caused by an identical locus. The physical positions of the associations for the $\alpha$ - and $\beta$-carotene contents on chromosome 3 were close, and the highest associations were detected at a physical position around 6 $\mathrm{Mb}$ (Fig. 3, Table 1). An association detected in population B for visual color evaluation on chromosome 3 showed the highest association at physical position $5.4 \mathrm{Mb}$, and this physical position was similar to those of the associations detected in population A for $\alpha$ - and $\beta$-carotene contents (Figs. 3, 4, Table 1). These results suggest that the associations are caused by an identical locus. Interestingly, the association detected on chromosome 5 (showing the highest association for visual evaluation in $\mathrm{F}_{2}$ population A) was not detected in any other evaluations (Fig. 3, Table 1). 
Table 1

Significant associations for carrot root color identified by GWAS

\section{Trait}

Visual evaluation

$b^{*}$

a-carotene

Lutein

$\beta / a-c a r o t e n e$ ratio

$a^{\star}$

$\beta$-carotene

F2_population

physical position $^{a}$

$-\log 10 P$

Chr bp

\begin{tabular}{llll} 
A & 5 & $39,247,011$ & 5.80 \\
A & 1 & $30,782,032$ & 5.31 \\
\hline A & 4 & $20,589,731$ & 5.01 \\
\hline A & 6 & $6,440,506$ & 4.72 \\
B & 3 & $5,419,538$ & 9.69
\end{tabular}

A

1

A 3

A

$A$

A

A

A

A

A

A

A

A 2

A

$\mathrm{A}$

A

A

A

$32,693,618 \quad 8.42$

$3 \quad 1,689,065 \quad 6.37$

1

$31,112,534$

12.49

1

$30,704,558$

11.08

$15,832,013$

8.22

$5,849,853$

7.77

$30,704,558$

13.72

A

A




\section{Correlations among visual evaluation, color components, and carotene contents in root of $F_{2}$ population $A$}

The Pearson correlation between each phenotype showed that three color components, i.e., $L^{*}, a^{*}$ and $b^{*}$, the a-carotene content, and the $\beta$-carotene content were highly correlated (Table 2$)$. The lutein content was slightly correlated with $L^{*}, a^{*}$ and $b^{*}$ and highly correlated with the a-carotene content. As lutein is biosynthesized downstream of the a-carotene (Fig. 1), this high correlation of lutein and a-carotene is consistent with the biosynthesis pathway. The visual evaluation was not highly correlated with any other phenotypes.

\section{Allelic effects of associations detected by GWAS on chromosomes 1 and 3 for the a-carotene and $\beta$-carotene contents in $F_{2}$ population $A$}

We examined the allelic effects of the associations detected by the GWAS for the $\alpha$ - and $\beta$-carotene contents. At the median, the carrots with AA allele on the SNP showing the highest association for $a-$ carotene (DCARV2_CHR1_30704558) had approx. 1.5-fold higher contents of $\alpha$ - and $\beta$-carotene than those with GG allele (Fig. 5a, b). Similarly, at the median, the carrots with GG allele on the SNP showing the highest association for a-carotene (DCARV2_CHR3_5849853) had approx. 1.3-fold higher contents of a-carotene and approx. 1.2-fold higher contents of $\beta$-carotene compared to those with AA allele (Fig. 5c, d). A clear genetic interaction such as epistasis was not observed between the associations detected on chromosomes 1 and 3 (Suppl. Fig. S2). Together with both associations detected on chromosomes 1 and 3 , at the median, the carrots that had alleles showing higher carotenoid content in both associations also had approx. 2.6-fold higher a-carotene and approx. 1.8-fold higher $\beta$-carotene contents in carrot surface compared to those with alleles showing lower carotenoid contents in both associations (Suppl. Fig. S2).

\section{Possible candidate gene for the association detected on chromosome 3 by the GWAS and sequence comparison between parents in $F_{2}$ populations $A$ and $B$}

Significant associations were detected around the physical position at 5-6 Mb on chromosome 3 for acarotene and $\beta$-carotene contents in $F_{2}$ population $A$ and for visual evaluation in $F_{2}$ population $B$ (Figs. 3 , 4, Table 1). Within this region, the reported Orgene (DCAR_009172), which affects carotenoid contents in carrot [16], is located at $5.2 \mathrm{Mb}$ on chromosome 3. To examine the involvement of Or, we performed Sanger sequencing of Or in the parents of populations A and B. The Sanger sequencing detected only one non-synonymous amino acid substitution at the fourth amino acid from the end, which was caused by an SNP between both parents of $F_{2}$ populations $A$ and $B$ (Fig. 6a). A thymine which was identical to that in 
the carrot reference genome [10] in Fs001 and Fs003 was changed to guanine in Fs002, which resulted in a change from Tyr309 in the Fs001 and Fs003 to aspartic acid in the Fs002.

To examine the effect of the SNP on Or causing the non-synonymous amino acid substitution, we developed a KASP marker which could genotype the SNP. We applied the developed KASP marker to breeding line $\mathrm{C}$ whose root color was segregated and that is the progeny of Fs002 (Fig. 2). The root color of breeding line $\mathrm{C}$ was visually evaluated into three grades (Fig. 6c). The genotype of KASP marker on Or was clearly correlated with the visual evaluation (Fig. 6b). All of the carrots whose root color was bright and middle orange had a heterozygote for the SNP on Or, and all of the carrots whose root color was slightly light orange had a TT homozygote for the SNP. We thus speculate that the associations detected in $F_{2}$ population $A$ for $\alpha$-carotene and $\beta$-carotene contents on chromosome 3 and the association detected in $F_{2}$ population $B$ for visual evaluation were responsible for the SNP causing the non-synonymous amino acid substitution on Or.

\section{Possible candidate gene for the $\beta / \alpha$-carotene ratio in population $A$, and the amino acid comparison between parents of population $A$}

In the GWAS of $F_{2}$ population $A$, the association for the $\beta / a$-carotene ratio was detected on chromosome 6 and showed the highest association on the physical position at around $4.6 \mathrm{Mb}$. lorizzo et al. [10] summarized the carrot orthologous and homologous candidate genes involved in the plastid 2-C-methylD-erythritol 4-phosphate (MEP) and carotenoid pathways in a table. According to the table, DCAR_022896 (which has a lycopene cyclase domain) is located on a physical position at $4.1 \mathrm{Mb}$ on chromosome 6, which is between the SNP showing the highest association for the $\beta / a$-carotene ratio and the next SNP (Suppl. Table S2). Carotenoid biosynthesis bifurcates after lycopene to produce e- and $\beta$-carotenoids by enzymatic activity of the two lycopene cyclases, lycopene e-cyclase (LCYE) and lycopene $\beta$-cyclase (LCYB) [28] (Fig. 1). In addition, it is known that the proportions of $\beta$-carotene and a-carotene are determined mostly by the comparative amounts and/or activities of the LCYB and LCYE enzymes [20, 29-32].

However, the genes annotated as carrot $\angle C Y B$ and $L C Y E$ are not DCAR_022896 [10, 24]. Our BLAST search (NCBl; http://www.ncbi.nlm.nih.gov) of amino acids of DCAR_022896 showed sequence homology with neoxanthin synthase (NYS) and capsanthin-capsorubin synthase (CCS). The Phytozome database annotated DCAR_022896 as NYS, CCS, and lycopene cyclase (https://phytozome.jgi.doe.gov/pz/portal.html\#). It is known that LCYB, NSY (which catalyzes violaxanthin into neoxanthin), CCS (which catalyzes the conversion of antheraxanthin and violaxanthin into capsanthin and capsorubin, respectively) (Fig. 1), and chromoplast-specific lycopene $\beta$-cyclase (CYCB) have high sequence homology and similar putative catalytic mechanisms $[20,21,33,34]$. 
Our phylogenic analysis of DCAR_022896, LCYE, LCYB, NSY, CCS, and CYC-B in carrot and Arabidopsis as well as Solanum lycopersicum, Carica papaya, Citrus sinensis, Capsicum annuum, and Lillium lancifolium showed that DCAR_022896 belonged to the same clade as CYC-B in C. sinensis and C. papaya (Fig. 7a). At the amino acid level, DCAR_022896 had $76.9 \%$ identity to CYC-B in C. sinensis and $62.1 \%$ to CYC-B in C. papaya. CYC-B is a $\angle C Y B$, and it converts lycopene to $\beta$-carotene in chromoplasts, where carotenoids are accumulated [35, 36], in a specific manner [20] (Fig. 1). Moreover, our BLAST search of primers for the reported $\angle C Y B 2$ in carrot showed that CYC-B (DCAR_022896) in the present study is identical to $L C Y B 2[5,6,37,38]$. We thus presume that DCAR_022896 is a possible candidate gene for the $\beta / \alpha$-carotene ratio, and we compared the amino acid sequences between the parents of $F_{2}$ population $A$ by Sanger sequencing. The amino acid comparison revealed five amino acid substitutions between the parents of $F_{2}$ population $A$ (Fig. $7 \mathrm{~b}$ ). These results suggested the possibility of the involvement of CYC-B in the $\beta / \alpha$-carotene ratio in carrot root.

\section{Discussion}

Our GWAS using the two $F_{2}$ populations derived from orange root carrots detected 21 associations for visual color evaluation, color component $a^{*}$ and $b^{*}, a$ - and $\beta$-carotene content, lutein content, and the $\beta / a-$ carotene ratio (Figs. 3, 4, Table 1). Some associations were detected on close physical positions for several evaluations of root color. However, interestingly, associations for visual evaluation in $F_{2}$ population A on chromosomes 4 and 5 were not detected for any other phenotypes. The Pearson correlation also showed no high correlation between visual color evaluation and other phenotypes (Table 2). These results suggest that we could not evaluate carrot root colors as same as experienced breeders by using spectrocolorimetry and HPLC. Experienced breeders evaluate root color comprehensively including the gloss and texture of the carrot surface, and thus the detected associations only for visual evaluation might be associated for these phenotypes. 


\section{Table 2}

Pearson correlation between visual evaluation, color components and carotenoid contents of carrot root in $\mathrm{F} 2$ population $\mathrm{A}$.

\begin{tabular}{|c|c|c|c|c|c|c|}
\hline & Visual evaluation & $L^{*}$ & $a^{*}$ & $b^{*}$ & a-carotene & $\beta$-carotene \\
\hline$L^{*}$ & 0.079 & & & & & \\
\hline$a^{*}$ & 0.093 & $0.674^{\star \star \star}$ & & & & \\
\hline$b^{*}$ & $-0.167^{*}$ & $0.615^{\star \star \star}$ & $0.899^{\star \star \star}$ & & & \\
\hline a-carotene & $-0.176^{*}$ & $0.367^{\star \star \star}$ & $0.533^{\star \star \star}$ & $0.537^{\star \star}$ & & \\
\hline$\beta$-carotene & 0.048 & $0.333^{\star \star \star}$ & $0.547^{\star \star \star}$ & $0.508^{\star \star}$ & $0.775^{\star \star *}$ & \\
\hline lutein & 0.065 & $0.266^{*}$ & $0.265^{*}$ & $0.186^{*}$ & $0.354^{\star \star \star}$ & $0.284^{\star \star}$ \\
\hline
\end{tabular}

On the other hand, the associations detected herein on chromosome 1 were significant for visual evaluation, color components $a^{*}$ and $b^{*}$, and the $a$ - and $\beta$-carotene contents (Fig. 3, Table 1). However, there are no annotated genes for MEP and carotenoid pathways within $5 \mathrm{Mb}$ from the physical position of $31 \mathrm{Mb}$ on chromosome 1 where the highest association was detected [10]. Similarly, a highly significant association was detected in $\mathrm{F}_{2}$ population $\mathrm{A}$ for lutein content on chromosome 5 (Fig. 3, Table 1), whereas there are no predicted genes annotated for MEP and carotenoid pathways [10] or for chromatin-modifying histone methyltransferase, SDG8 (CCR1), which affects the lutein content in leaves [39] around this locus except for neoxanthin synthase (NSY). The NSY gene is located approx. 1.1 Mb away from the physical position of the highest association for lutein content. NSY has a role downstream of another branch which does not include lutein in carotenoid biosynthesis (Fig. 1), and no feedback regulation between NSY and lutein content has been reported. However, we cannot exclude the possibility that the mutation of the NSY of another branch affects the flow rate of each branch, resulting in an effect on the lutein content. Further analyses such as a map-based strategy is necessary to narrow down the candidate regions and identify candidate genes causing the associations detected on chromosome 1 for visual evaluation, color components $a^{*}$ and $b *$, and $a$ - and $\beta$-carotene contents and chromosome 5 for lutein content, and for the other significant associations revealed in this study.

The GWAS in $F_{2}$ population $A$ for the $\alpha$ - and $\beta$-carotene content and in $F_{2}$ population $B$ for visual evaluation detected the association at a similar physical position around 5-6 $\mathrm{Mb}$ on chromosome 3 (Figs. 3, 4, Table 1), and the previously reported Orwas located on a similar physical position (5.2 Mb). The Orgene is involved in carotenoid accumulation via chromoplast development and biosynthesis via 15-cis-phytoene synthase (PSY) expression, and it affects the total carotenoid content [40, 41]. Carrot Or was recently identified and is associated with the carotenoid presence in carrot root [16]. The similarity of 
physical positions and function of Or suggests that these associations were caused by $O r$, in addition, an SNP causing a non-synonymous mutation was detected in the present study between the parents of $F_{2}$ populations A and B by Sanger sequencing (Fig. 6a). lorizzo et al. [10] re-sequenced 35 carrot accessions and released SNPs on Phytozome (https://phytozome.jgi.doe.gov/pz/portal.html). In the released SNPs, there are many SNPs on the Orgene, including three SNPs causing a non-synonymous mutation. However, the SNP that causes the non-synonymous mutation detected in Fs002 is not included, suggesting that this is a new allele of Orand that a distribution of the SNP detected in this study might be limited. Further analyses of the distribution of this SNP are needed. As breeding line $C$ was derived from Fs002 (Fig. 2), a bright orange allele would be derived from the Fs002. Peaks of the Manhattan plots of this region were also observed for visual color evaluation (Fig. 3a), color components $L^{*}$ (Fig. $3 b$ ) and b* (Fig. 3d), and lutein content (Fig. 3g). Although their associations were not significant, these peaks might be caused by $O r$.

The GWAS in $F_{2}$ population A revealed the association for the $\beta /$ a-carotene ratio on chromosome $6 ; \mathrm{CYC}$ $B$ is located on the associated region (Fig. 3h, Suppl. Table S2). In several model plants such as Arabidopsis thaliana [42], rice (Oryza sativa) [43], and maize (Zea mays) [29, 44], LCYB is encoded by a single gene. However, LCYB is encoded by two genes in some plant species that accumulate high levels of carotenoids in non-photosynthetic organs, such as fruits and flowers [38]. These genes are differentially expressed in photosynthetic and non-photosynthetic organs, and genes that are expressed in non-photosynthetic organs were named $C Y C-B$. As named, $C Y C-B$ is a chromoplast-specific lycopene $\beta-$ cyclase.

Carrot has two LCYBs: LCYB1 and LCYB2 [3]. Our present phylogenetic analysis demonstrated that carrot LCYB1 (LCYB) and LCYB2 (CYC-B) belong to LCYB and CYC-B clades, respectively (Fig. 7a). The CYC-B was first reported in tomato (Solanum lycopersicum) as a fruit- and flower-specific lycopene $\beta$-cyclase using two mutations named Beta and old-gold. Beta increases the $\beta$-carotene content in fruit, and oldgold, a null mutation of $C Y C-B$, abolishes $\beta$-carotene and increases lycopene contents in fruits and caused tawny orange flowers [20]. The $C Y C-B$ has also been reported to be responsible for fruit color in papaya (Carica papaya) [22] and citrus (Citrus sinensis) and for the involvement of the null allele in the high lycopene accumulation in red grapefruits [23]. In carrot, unlike plants that have organ-specific LCYBs, $\angle C Y B 1$ is expressed in both leaves and root, and the transcript level of $L C Y B 1$ increases as the carotenoid content increases during root development $[38,45]$. Since the GWAS detected an association around the $C Y C-B$ region in this study, we speculate that in carotenoid-accumulating carrot root, in addition to the LCYB (LCYB1), CYC-B (LCYB2) would also have a role in carotenoid biosynthesis. To the best of our knowledge, the involvement of $C Y C-B(L C Y B 2)$ in carotenoid contents in carrot root has not been examined. A chromoplast-specific carotenoid biosynthesis pathway including $C Y C-B$ such as that reported in tomato [46] might have a role in carrot roots. Further functional analyses such as an expression study of $C Y C-B(\angle C Y B 2)$ in several organs and an investigation of the subcellular location of CYC-B (LCYB2) in carrot are necessary to clarify the functions of the two LCYBs. 
Visual appearance traits are important targets in carrot breeding in Japan, and the 'best bright orange color' is selected based on a comparison of minute color differences as shown in Figure $6 \mathrm{c}$. The differences are actually difficult for non-specialists to detect, but the resultant selected cultivars attract consumers in Japan with their 'best bright orange color'. The present study provides the first results of a GWAS analysis for carrot root color for the selection of bright orange color in orange root populations. The developed KASP marker on Or as well as the SNPs showing significant associations will contribute to orange carrot breeding.

\section{Declarations}

\section{Data availability}

Nucleotide sequence data for the ddRADseq in $F_{2}$ population $A$ and $B$ is available in the DDBJ Sequence Read Archive under accession numbers from DRA012848 to DRA012853.

\section{Acknowledgements}

We are grateful to Dr. K. Shirasawa at the Kazusa DNA Research Institute for technical advises, and Ms. S. Sasamoto, Ms. R. Aomiya, and Ms. T. Shibazaki at the Kazusa DNA Research Institute for their technical assistance. We are also grateful to Mr. H. Hirose, and Mr. Y. Yoshida and Mr. S. Yoshida at Yoshida Seed for their carrot-field management, and to all staff members at Fujii Seed for preparing carrot materials.

\section{Author contributions}

T.S. designed the experiment, performed GWAS, and wrote the manuscript; S.I. provided direction for the study, designed the experiment, and correction to the manuscript; H.T., C.M. and A.O. performed the experiment; S.N. performed NGS data analysis; C.K. and T.F. provided plant materials and evaluated phenotypes. All authors read and approved the final manuscript.

\section{Competing Interests:}

The authors declare no competing interests.

\section{References}

1. Simon, P., Pollak, L., Clevidence, B., Holden, J. \& Haytowitz, D. Plant breeding for human nutrition. Plant Breed. Rev. 31, 325-392 (2009). 
2. Krinsky, N. I., Johnson, E. J. Carotenoid actions and their relation to health and disease. Mol. Aspects Med. 26, 459-516 (2005).

3. Just, B. J., Santos, C. A. F., Fonseca, M. E. N., Boiteux, L. S., Oloizia, B. B., \& Simon, P. W. Carotenoid biosynthesis structural genes in carrot (Daucus carota): Isolation, sequence-characterization, single nucleotide polymorphism (SNP) markers and genome mapping. Theor. Appl. Genet. 114, 693-704. doi: 10.1007/s00122-006-0469-x. (2007).

4. Santos, C. A. F. \& Simon, P. W. QTL analyses reveal clustered loci for accumulation of major provitamin A carotenes and lycopene in carrot roots. Mol. Genet. Genomics. 268, 122-129 (2002).

5. Just, B. J., Santos, C. A. F., Yandell, B. S. \& Simon, P. W. Major QTL for carrot color are positionally associated with carotenoid biosynthetic genes and interact epistatically in a domesticated $\times$ wild carrot cross. Theor. Appl. Genet. 119, 1155-1169 (2009).

6. Jourdan, M., et al. Carotenoid content and root color of cultivated carrot: A candidate-gene association study using an original broad unstructured population. PLOS ONE. 10, e0116674. doi: 10.1371/journal.pone.0116674 (2015).

7. Ruiz-Sola, M. Á. \& Rodríguez-Concepción M. Carotenoid biosynthesis in Arabidopsis: A colorful pathway. Arabidopsis Book. 10, e0158. doi: 10.1199/tab.0158 (2012).

8. Stanley, L., Yuan, Y.-W. Transcriptional regulation of carotenoid biosynthesis in plants: so many regulators, so little consensus. Front. Plant Sci. 10, 1017. doi: 10.3389/fpls.2019.01017 (2019).

9. Al-Babili, S. et al. Identification of a novel gene coding for neoxanthin synthase from Solanum tuberosum. FEBS Lett. 485, 168-172 (2000).

10. Iorizzo, M. et al. A high-quality carrot genome assembly provides new insights into carotenoid accumulation and asterid genome evolution. Nat. Genet. 48, 657-666 (2016).

11. Wang, F., Wang, G.-L., Hou, X.-L., Li, M.-Y., Xu, Z.-S. \& Xiong, A.-S. The genome sequence of 'Kurodagosun', a major carrot variety in Japan and China, reveals insights into biological research and carrot breeding. Mol. Genet. Genomics. 293, 861-871 (2018).

12. Arango, J., Jourdan, M., Geoffriau, E., Beyer, P. \& Welsch, R. Carotene hydroxylase activity determines the levels of both a-carotene and total carotenoids in orange carrots. Plant Cell. 26, 2223-2233 (2014).

13. Buishand, J. \& Gabelman, W. Investigations on the inheritance of color and carotenoid content in phloem and xylem of carrot roots (Daucus carota L.). Euphytica 28, 611-632 (1979).

14. Ellison, S., Senalik, D. Bostan, H, lorizzo, M. \& Simon, P. Fine mapping, transcriptome analysis, and marker development for $\mathrm{Y} 2$, the gene that conditions $\beta$-carotene accumulation in carrot (Daucus carota L.). G3 (Bethesda). 7, 2665-2675. doi: 10.1534/g3.117.043067 (2017).

15. Li, L., Paolillo, D., Parthasarathy, M., Dimuzio, E. \& Garvin, D. A novel gene mutation that confers abnormal patterns of $\beta$-carotene accumulation in cauliflower (Brassica oleracea var. Botrytis). Plant J. 26, 59-67 (2001).

16. Ellison, S. L. et al. Carotenoid presence is associated with Orgene in domesticated carrot. Genetics. 210, 1497-1508 (2018). 
17. Shirasawa, K., Hirakawa, H., \& Isobe, S. Analytical workflow of double-digest restriction siteassociated DNA sequencing based on empirical and in silico optimization in tomato. DNA Res. 23, 145-153 (2016).

18. Arafa, R. A. et al. Rapid identification of candidate genes for resistance to tomato late blight disease using next-generation sequencing technologies. PLOS ONE. 12, e0189951. doi: 10.1371/journal.pone.0189951 (2017).

19. Bradbury, P. J. et al. TASSEL: Software for association mapping of complex traits in diverse samples. Bioinformatics. 23, 2633-5 (2007).

20. Ronen, G., Carmel-Goren, L., Zamir, D., \& Hirschberg J. An alternative pathway to $\beta$-carotene formation in plant chromoplasts discovered by map-based cloning of Beta and old-gold color mutations in tomato. Proc. Natl. Acad. Sci. 97, 11102-11107 (2000).

21. Jeknić, Z. et al. Cloning and functional characterization of a gene for capsanthin-capsorubin synthase from tiger lily (Lilium lancifolium Thunb. 'Splendens'). Plant Cell Physiol. 53, 1899-1912. doi: 10.1093/pcp/pcs128 (2012).

22. Blas, A. L. et al. Cloning of the papaya chromoplast-specific lycopene b-cyclase, CpCYC-b, controlling fruit flesh color reveals conserved microsynteny and a recombination hot spot. Plant Physiol. 152, 2013-2022. doi: 10.1104/pp.109.152298 (2010)

23. Alquézar, B., Zacarías, L., Rodrigo, M. J. Molecular and functional characterization of a novel chromoplast-specific lycopene b-cyclase from Citrus and its relation to lycopene accumulation. J. Exp. Bot. 60, 1783-1797 (2009).

24. Simon, P.W., Geoffriau, E., Ellison, S., \& Iorizzo, M. Carrot carotenoid genetics and genomics, in The carrot genome (eds. Simon, P., lorizzo, M., Grzebelus, D., Baranski, R.) 247-260 (Springer, 2019).

25. Thompson, J. D., Higgins, D. G., \& Gibson, T. J. CLUSTAL W: Improving the sensitivity of progressive multiple sequence alignment through sequence weighting, position-specific gap penalties and weight matrix choice. Nucleic Acids Res. 22, 4673-4680 (1994).

26. Saitou, N., \& Nei, M. The neighbor-joining method: A new method for reconstructing phylogenetic trees. Mol. Biol. Evol. 4, 406-425 (1987).

27. Kumar, S., Stecher, G., Li, M., Knyaz, C., \& Tamura, K. MEGA X: Molecular Evolutionary Genetics Analysis across computing platforms. Mol. Biol. Evol. 35, 1547-1549 (2018).

28. Cazzonelli, C. I, \& Pogson, B. J. Source to sink: Regulation of carotenoid biosynthesis in plants. Trends Plant. Sci. 15, 266-274 (2010).

29. Bai, L., Kim, E.-H., DellaPenna, D., Brutnell, T. P. Novel lycopene epsilon cyclase activities in maize revealed through perturbation of carotenoid biosynthesis. The Plant J. 59, 588-599 (2000).

30. Pogson, B., McDonald, K. A., Truong, M., Britton, G., DellaPenna, D. Arabidopsis carotenoid mutants demonstrate that lutein is not essential for photosynthesis in higher plants. Plant Cell. 8, 1627-1639 (1996).

31. Ronen, G., Cohen, M., Zamir, D., Hirschberg, J. Regulation of carotenoid biosynthesis during tomato fruit development: Expression of the gene for lycopene epsilon-cyclase is down-regulated during 
ripening and is elevated in the mutant Delta. The Plant J. 17, 341-351 (1999).

32. Harjes, C. E. et al. Natural genetic variation in lycopene epsilon cyclase tapped for maize biofortification. Science. 319, 330-333. doi: 10.1126/science.1150255 (2008).

33. Bouvier, F., d'Harlingue, A., Backhous, R. A., Kumagai, M. H. \& Camara, B. Identification of neoxanthin synthase as a carotenoid cyclase paralog. Eur. J. Biochem. 267, 6346-6352 (2000).

34. Mialoundama, A. S. et al. Characterization of plant carotenoid cyclases as members of the flavoprotein family functioning with no net redox change. Plant Physiol. 153, 970-979 (2010).

35. Egea, I. et al. Chromoplast differentiation: current status and perspectives. Plant Cell Physiol. 51, $.1601-1611$ (2010).

36. Kim, J. E., Rensing, K. H., Douglas, C. J., Cheng, K. M. Chromoplasts ultrastructure and estimated carotene content in root secondary phloem of different carrot varieties. Planta. 231, 549-558 (2010).

37. Fuentes, P., Pizarro, L., Moreno, J. C., Handford, M., Rodriguez-Concepcion, M., Stange, C. Lightdependent changes in plastid differentiation influence carotenoid gene expression and accumulation in carrot roots. Plant Mol. Biol. 79, 47-59 (2012).

38. Moreno, J. C., Pizarro, L., Fuentes, P., Handford, M., Cifuentes, V., Stange, C. Levels of lycopene bcyclase 1 modulate carotenoid gene expression and accumulation in Daucus carota. PLOS ONE. 8, e58144. doi: 10.1371/journal.pone.0058144 (2013).

39. Cazzonelli, C. I. et al. Regulation of carotenoid composition and shoot branching in Arabidopsis by a chromatin modifying histone methyltransferase, SDG8. Plant Cell. 21, 39-53. doi: 10.1105/tpc.108.063131 (2009).

40. Lu, S. et al. The cauliflower Orgene encodes a DnaJ cysteine-rich domain containing protein that mediates high levels of $\beta$-carotene accumulation. The Plant Cell. 18, 3594-3605 (2006).

41. Zhou, X. et al. Arabidopsis OR proteins are the major posttranscriptional regulators of phytoene synthase in controlling carotenoid biosynthesis. Proc. Natl. Acad. Sci. USA. 112, 3558-3563 (2015),

42. Lange, B. M., Ghassemian, M. Genome organization in Arabidopsis thaliana: A survey for genes involved in isoprenoid and chlorophyll metabolism. Plant Mol. Biol. 51, 925-948 (2003).

43. Fang, J. et al. Mutations of genes in synthesis of the carotenoid precursors of ABA lead to preharvest sprouting and photo-oxidation in rice. Plant J. 54, 177-189 (2008).

44. Singh, M. et al. Activator mutagenesis of the pink scutellum1/viviparous7 locus of maize. Plant Cell. 15, 874-884 (2003).

45. Fuentes. P. et al. Light-dependent changes in plastid differentiation influence carotenoid gene expression and accumulation in carrot roots. Plant Mol. Biol. 79, 47-59. doi: 10.1007/s11103-0129893-2 (2012).

46. Galpaz, N., Ronen, G., Khalfa, Z., Zamir, D., Hirschberg, J. A chromoplast-specific carotenoid biosynthesis pathway is revealed by cloning of the tomato white-flower locus. Plant Cell. 18, 19471960 (2006). 
Figures

GGPP

$\downarrow P S Y$

Phytoene

$\downarrow$ PDS

$\downarrow Z-I S O$

$\downarrow$ ZDS

$\downarrow$ CRTISO

\section{Lycopene}

$\alpha$-carotene

LCYB $\downarrow$

$\delta$-carotene

CYP97A3 $\downarrow$

Zeinoxanthin

CYP97C1 $\downarrow$

\section{Lutein}

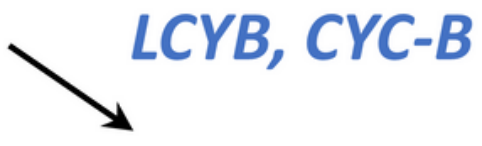

p-carotene $\downarrow \angle C Y B$

$\boldsymbol{\beta}$-carotene $\downarrow B C H$ $\beta$-cryptoxanthin

$\downarrow B C H$

Zeaxanthin

Capsanthin CCS Capsorubin $\downarrow$ ZEP

Antheraxanthin

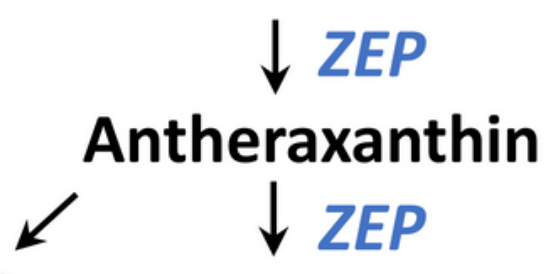

Violaxanthin

$\downarrow N S Y$

Neoxanthin

Figure 1

Carotenoid biosynthesis pathways. The carotenoid biosynthesis pathway is shown in black, with carotenoid biosynthesis genes indicated in blue. Figure compiled and summarized from Stanley et al. [8] and Al-Babili et al. [9]. 


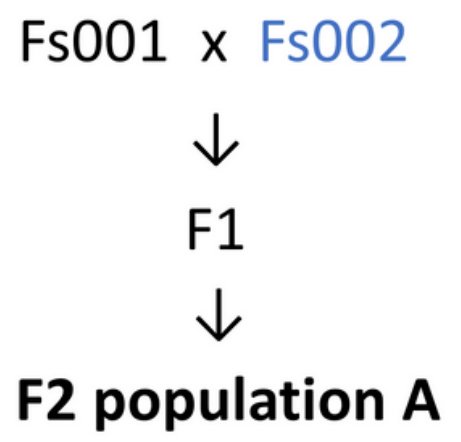

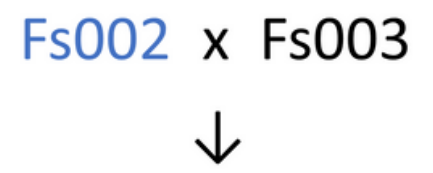

F1

$\downarrow$

F2 population B
$\mathrm{Fs002}$ x Fs004

$\downarrow$

$\mathrm{F} 1 \times \mathrm{Fs} 004$

$\downarrow$

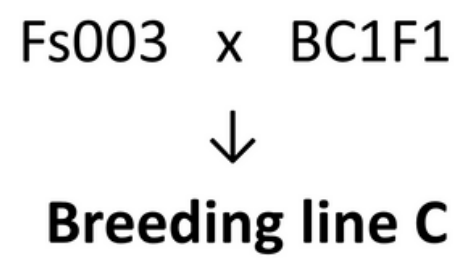

Figure 2

Lineage images of the plant materials, F2 populations A and B, and breeding line C. Fs002 was used as a common breeding material. 

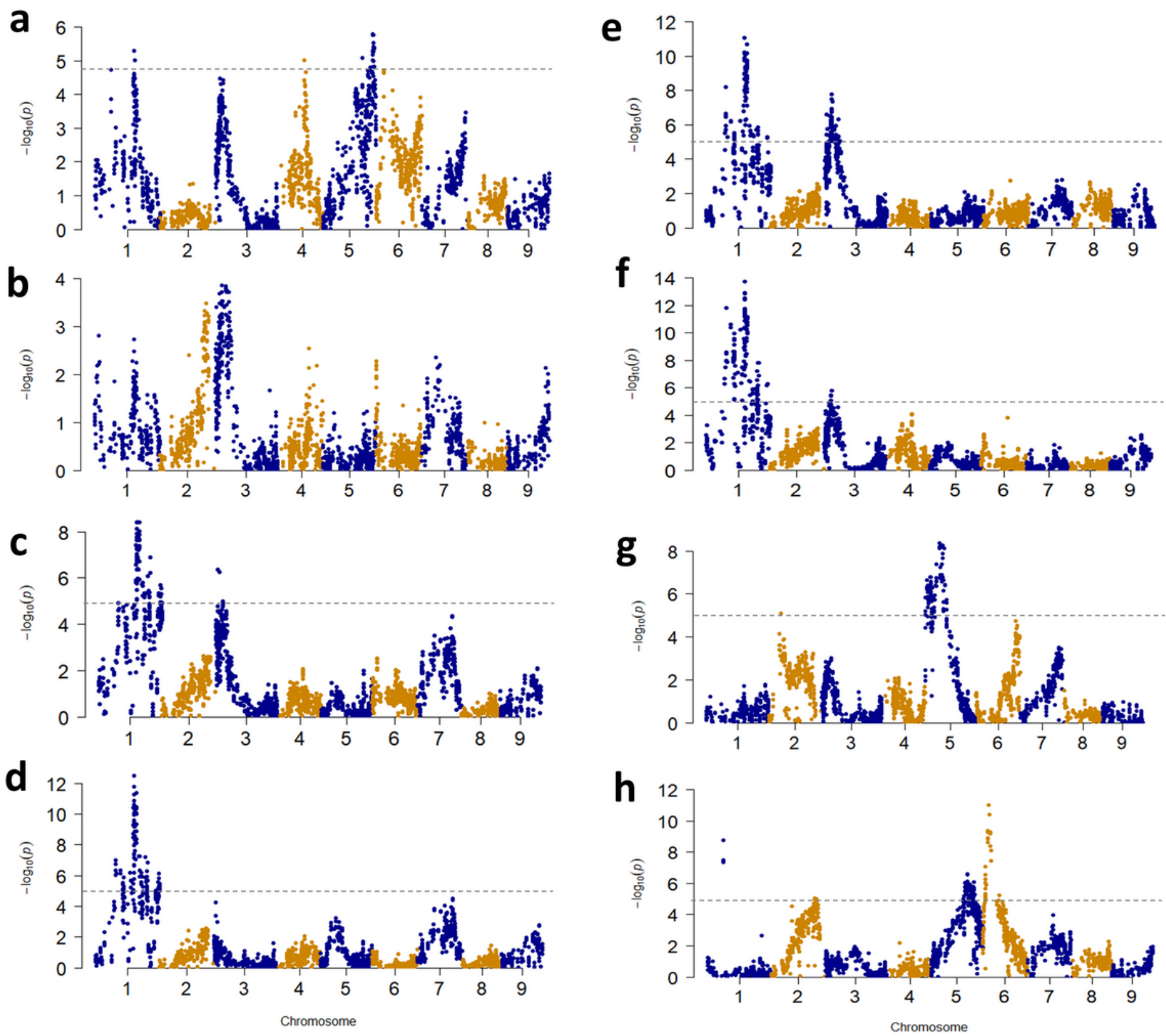

Figure 3

Manhattan plots for carrot taproot color in F2 population A. Plots for the visual evaluation (a), $L^{*}(b), a^{*}$ $(c), b^{\star}(d)$, a-carotene (e), $\beta$-carotene (f), lutein (g), and the $\beta$ / a-carotene ratio (h). Horizonal line indicates the Bonferroni correction (0.05). 


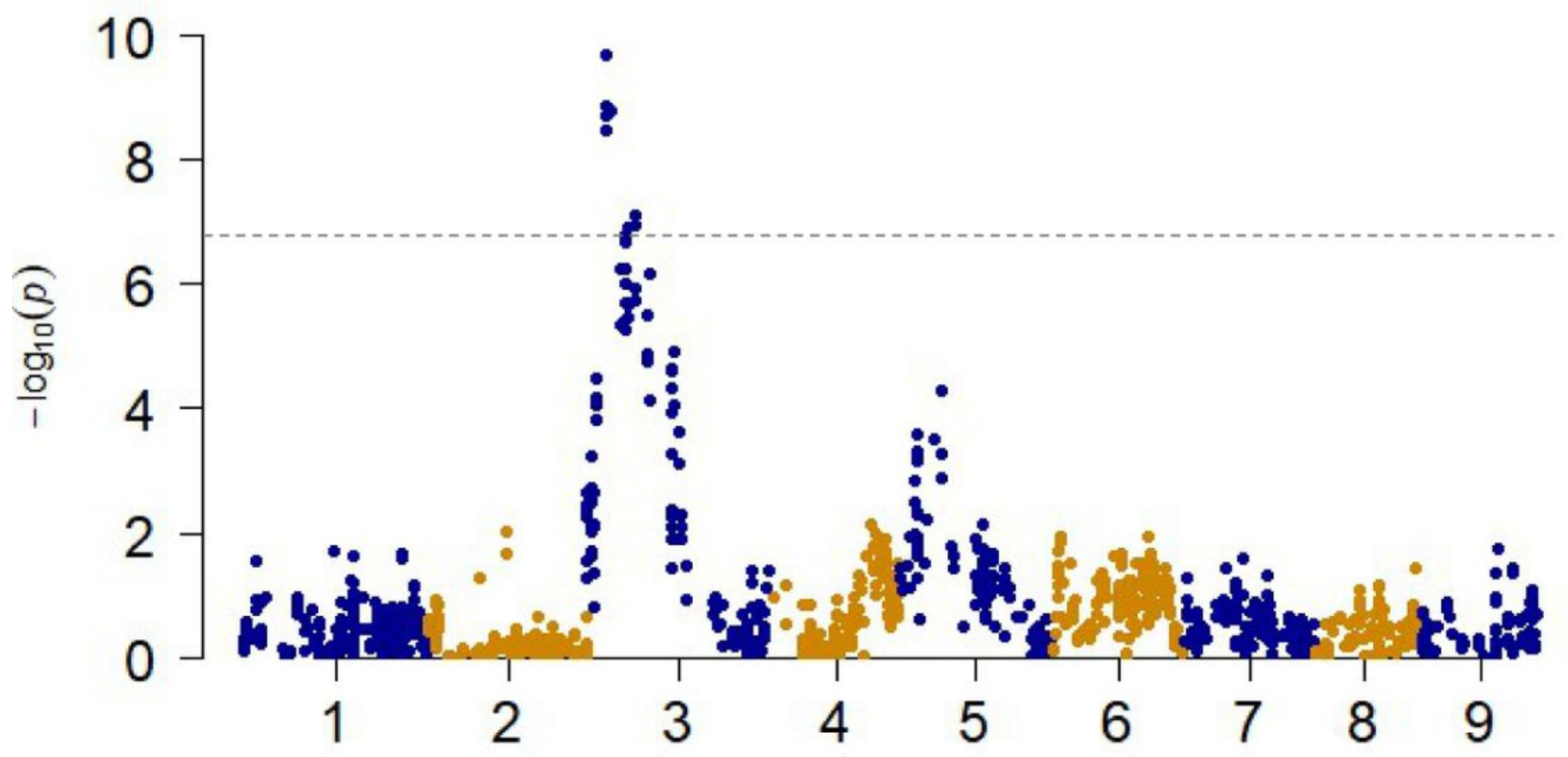

Chromosome

Figure 4

Manhattan plots for carrot taproot color in F2 population B. Plots for the visual evaluation of carrot root color. Horizonal line: Bonferroni correction (0.05). 

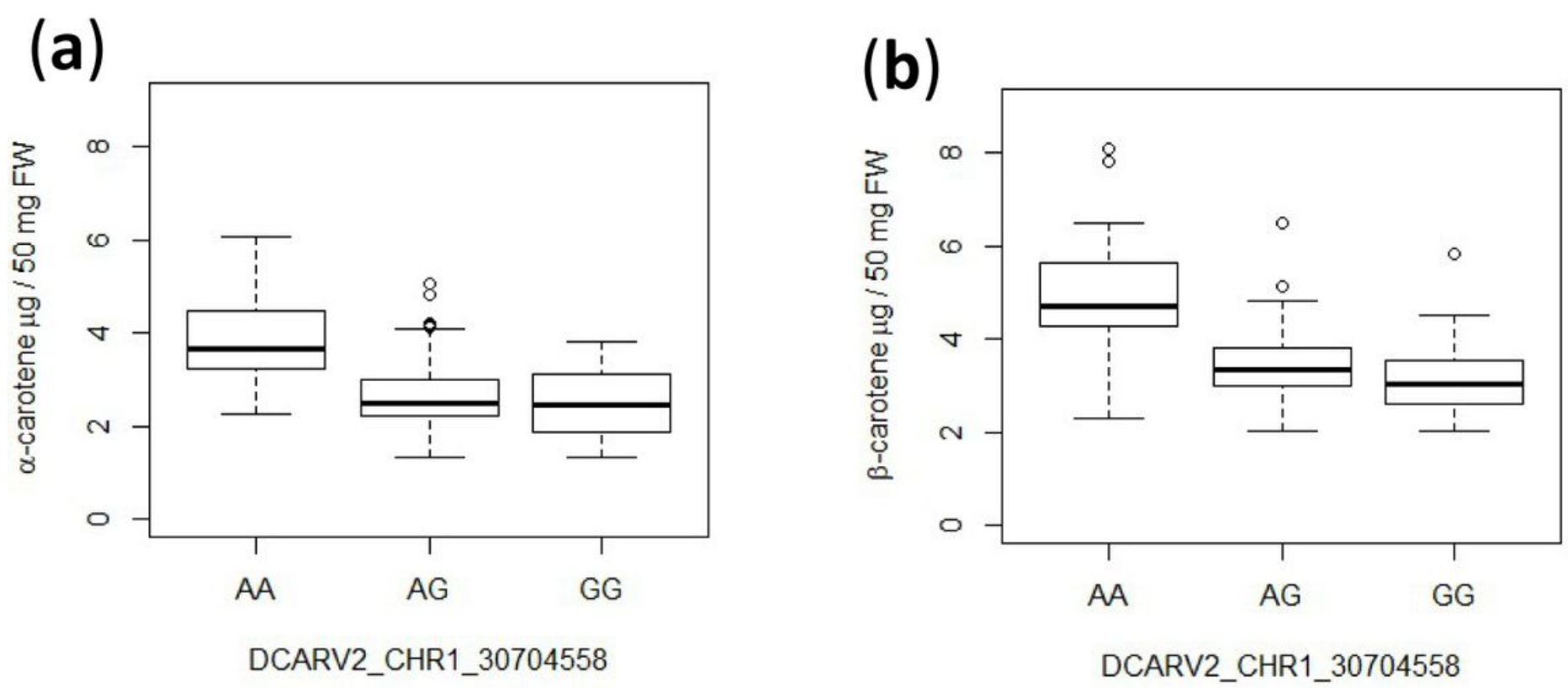

(c)

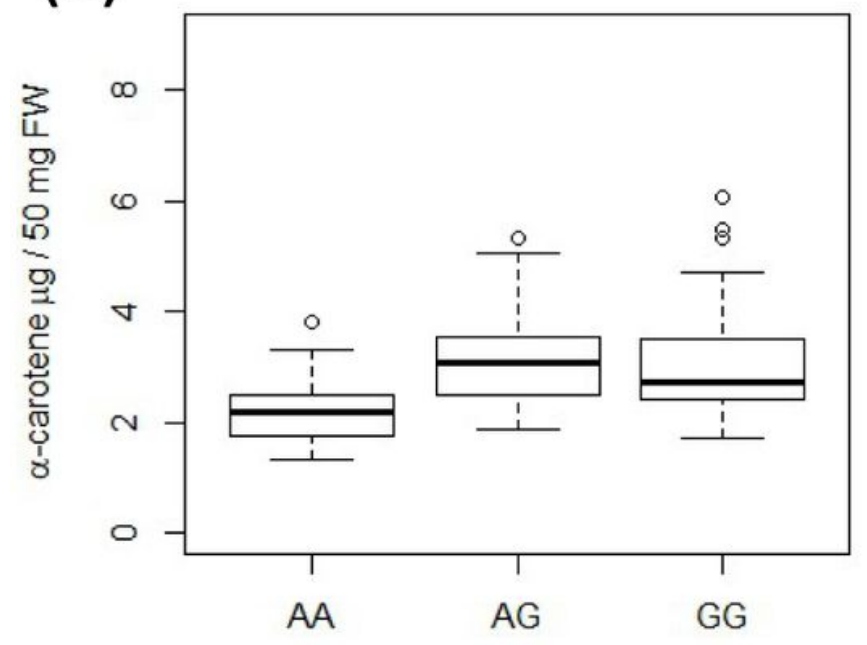

DCARV2_CHR3_5849853

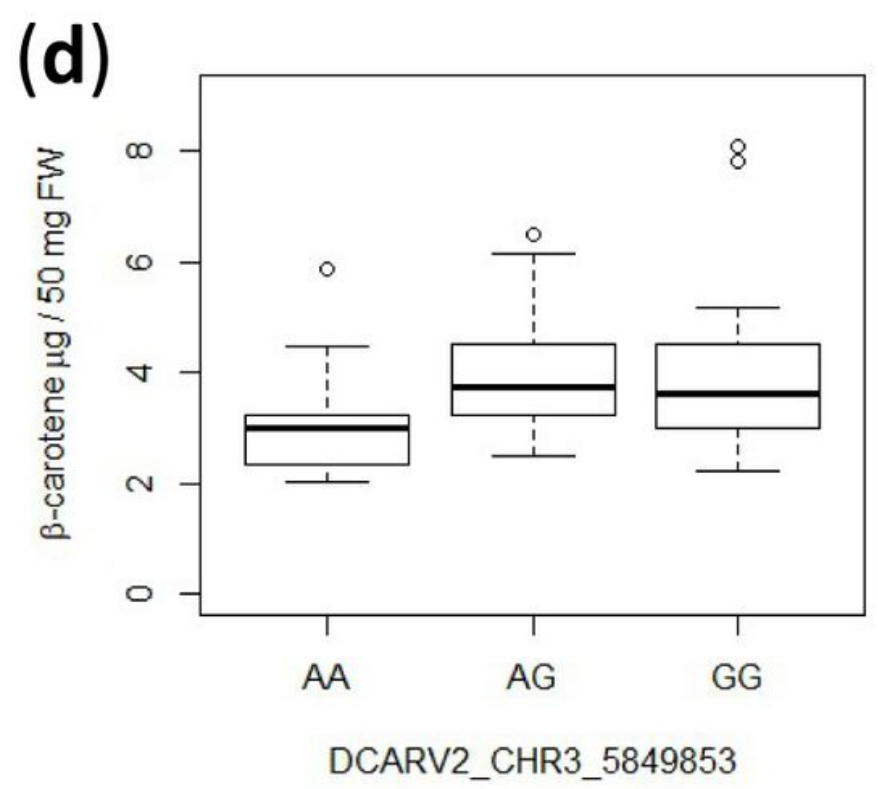

\section{Figure 5}

Allelic effects of associations detected by the GWAS on chromosome $1(a, b)$ and chromosome $3(c, d)$ for the $a$-carotene $(a, c)$ and $\beta$-carotene $(b, d)$ content in carrot root surface. Carotene contents were boxplotted by the SNP showing the highest - $\log 10 P$ in the GWAS for a-carotene content. The numbers of plants were 30, 81 and 34 for the AA, AG, and GG SNPs respectively in panels a and b, and 40, 70, 35 for the $A A, A G$ and $G G$ SNPs respectively in panels $c$ and $d$. 
(a)

$5154 \mathrm{bp}$

Fs001, Fs003

GAACATGACCCCCGGTTTTATCCTTTTGATTAA

$\mathrm{Tyr}$

stop

$\mathrm{Fs} 002$

GAACATGACCCCCGGTTTGATCCTTTTGATTAA

Asp

stop

(b)

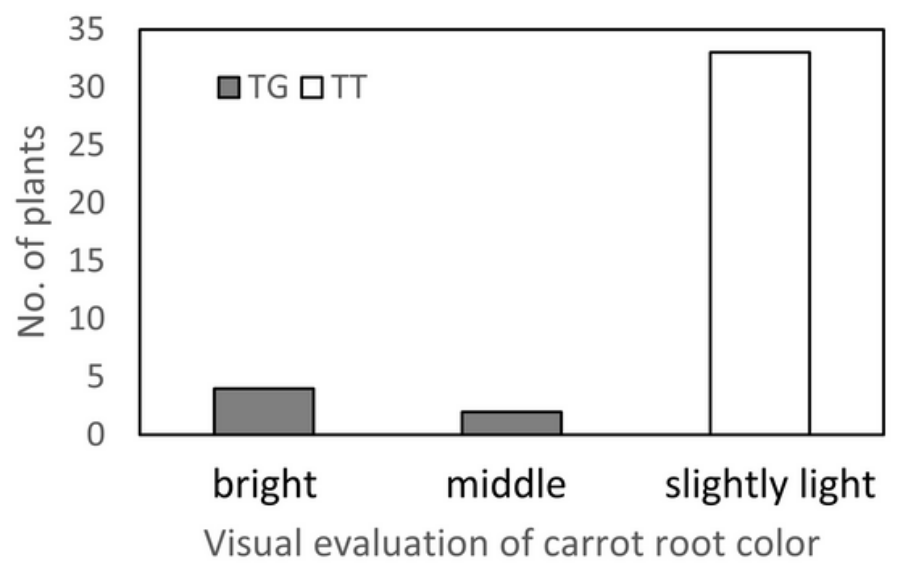

bright middle slightly light

(c)

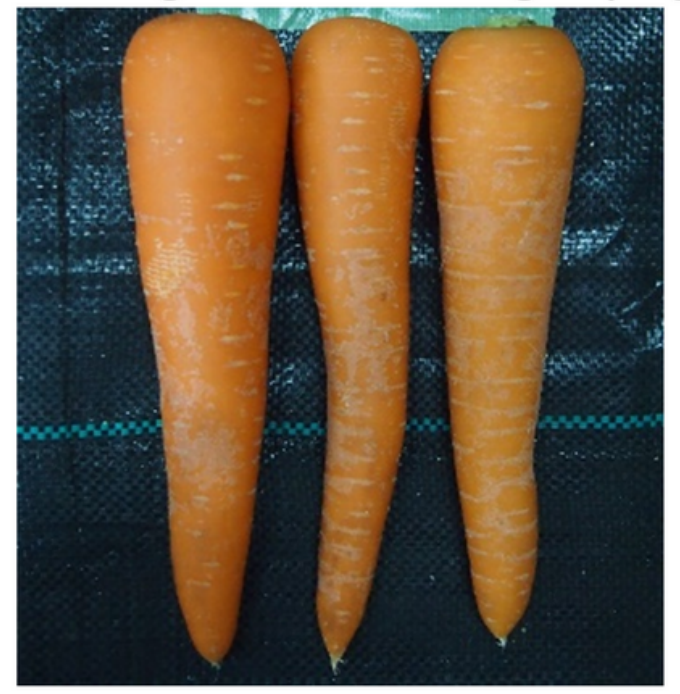

Figure 6

The SNP on Or and the examination of its effect on carrot root color in breeding line C. (a) The SNP detected on Or between parental plants in F2 populations A and B. The SNP causes an amino acid substitution. The upper sequence is identical to the reference sequence of lorizzo et al. [10], and the lower sequence is a new allele of Or. (b) The allelic effect of the SNP on Or in another breeding line at Fujii Seed. TT and TG showed TT homozygote and heterozygote of the Or SNP, respectively. Carrot root color was visually evaluated as three grades: brilliant, middle, and pale orange color $(b, c)$. All of the plants with bright or middle orange color roots showed a heterozygote for the Or SNP, and all plants with the slightly light orange color roots showed TT homozygote for the Or SNP. (c) Examples of carrot root color visually evaluated as three grades. 
(a)

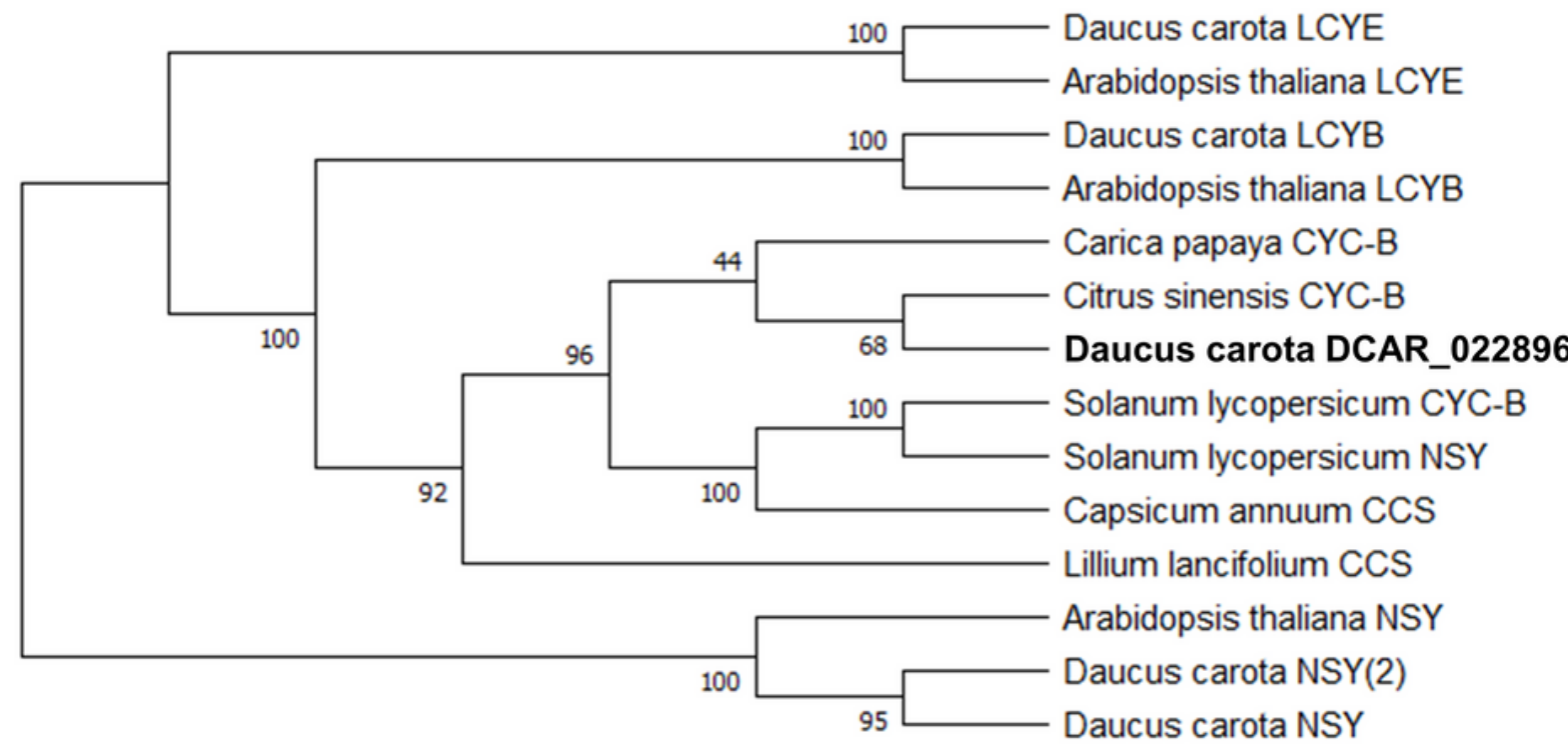

(b)

Fs001 METLKFIRPSSHPLIALHQSNYKA IKSPSLKYKPKKVTHTVQCSKYGNFLDLKPGKRHESMEFDLSWYDPSKRSRFDVIV Fs002 METLKFTRPSSHPSIALHQSNYKAVKSPSLKYKPKKVTHTVQCSKYGNFLDLKPGKRHESMEFDLSWYDPSKRSRFDVIV

Fs001 IGAGEAGLRIAERVAGYGICVCCVDPSPLCVWFNNYGVKVDEFEAMGFQDCFDKTWFMSSVY INEEKSKVINRPYGRVNR Fs002 IGAGEAGLRIAQRVAGYGICVCCVDPSPLCVWFNNYGVWVDEFEAMGFQDCFDKTWEMSSVYINEEKSKVINRPYGRVNR

$\begin{array}{llllllll}170 & 180 & 190 & 200 & 210 & 220 & 230 & 240\end{array}$

Fs001 EKLKMRLLGGCVSNGVVFHKAKVWKVDHQEFESSILCDDGKEFKASLIVDASGFASTFVDYDKPRNHGYQIAHGILAEVE Fs002 EKLKMRLLGGCVSNGVVFHKAKVWKVDHQEFESSILCDDGKEFKASLIVDASGFASTFVDYDKPRNHGYQIAHGIIAEVE

Fs001 SHPFELDRNVIMDWRDSHLGNEFALRF ANAKSPTFLYAMPFDSNLIFLEETSIVSREALSYKEVKLFNAARLRHLGIRVK

Fs002 SHPFELDRNVIMDWRDSHLGNEFALRF ANAKSPTFLYAMPFDSNLIFLEETSIVSREALSYKEVKLFNAARLRHLGIRVK

$\begin{array}{llllllll}330 & 340 & 350 & 360 & 370 & 380 & 390 & 400\end{array}$

Fs001 SIIEDEKCLIFMGGPLPRTPQDVVAIGGSSGIVHPSTGYNVARTIAIAFEIADAIAECLGSTRMIRGSSLYHRVKNGIWP

Fs002 SIIEDEKCLIFMGGPLPRTPODIVAIGGSSGIVHPSTGYNVARTIALAEVIADAIAECLGSTRMIRGSSLYHRVKNGIWP

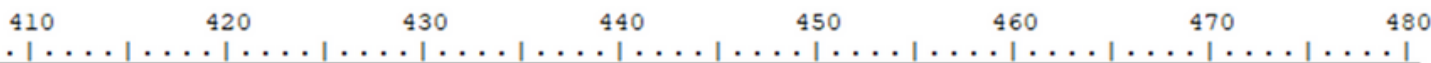

Fs001 IESKCTREFYSFGMETLLKLDINGTRNFFDAFFDLDPHYWQGFLSSRLSLKEI AMLSLSLFGHASNSSKMDIVTKCFAPL

Fs002 IESKCTREFYSFGMETLLKLDINGTRNFFDAFFDLDPHYWQGFLSSRLSLKEI AMLSLSLFGHASNSSKMDIVTKCFAPL

490

Fs001 VKMLGNIAVETI *

Fs002 VKMLGNIAVETI*

\section{Figure 7}

The phylogenetic tree based on the amino acid sequences of LCYE, LCYB, CYC-B, NSY, and CCS, and the amino acid substitutions between the parental plants of F2 population A on DCAR_022896. (a) The phylogenetic tree was drawn by the neighbor-joining method based on the amino acid sequences of Daucus carota DCAR_022896, D. carota LCYE (DCAR_028276), Arabidopsis thaliana LCYE (At5g57030), D. carota LCYB (DCAR_020544), A. thaliana LCYB (At3g10230), Solanum lycopersicum CYC-B, Carica 
papaya CYC-B (evm.model.supercontig_195.16), Citrus sinensis CYC-B (orange1.1g010693m.g), D. carota NSY (DCAR_017191), D. carota NSY(2) (DCAR_025914), A. thaliana NSY (At1g67080), S. lycopersicum NSY (CAB93342.1), Capsicum annuum CCS (Q42435.1), and Lillium lancifolium CCS (JF304153). Values at the nodes indicate the percentage consensus support as calculated using a bootstrapping test with 1,000 replications. (b) Five amino acid substitutions ( $)$ were detected between seed (Fs001) and pollen (Fs002) parents of F2 population A on DCAR_022896. Similar substitutions are shown in gray background.

\section{Supplementary Files}

This is a list of supplementary files associated with this preprint. Click to download.

- supplementaryfigures.pdf

- Supplementarytables.xlsx 